\title{
EXPRESSION OF ANTIMICROBIAL PEPTIDE GENES AFTER INFECTION BY PARASITOID WASPS IN Drosophila
}

\author{
Emmanuelle Nicolas, " Anthony J. Nappi† and Bruno Lemaitre* \\ "Institut de Biologie Moléculaire et Cellulaire, UPR 9022 du CNRS, 15 rue René Descartes, \\ 67084 Strasbourg, Cedex, France \\ †Department of Biology, Loyola University of Chicago, 6525 North Sheridan Road, \\ IL 60626, Chicago, U.S.A.
}

(Submitted 1 February 1996; Accecpted 1 April 1996)

\begin{abstract}
$\square$ Abstract-We report here the use of a specific $\beta$-galactosidase staining assay and Northern blotting technique to examine the expression of three genes encoding either antibacterial peptides (diptericin, cecropin A) or an antifungal peptide (drosomycin) in Drosophila following infection by larval and pupal parasitoids. The results show that the genes encoding these peptides are either not induced or minimally induced in waspinfected hosts, but remain responsive and are induced upon microbial challenge. As the parasitoids elicit a cellular response, our data suggest that the antimicrobial responses are activated and/or regulated by mechanisms that are independent of those mediating cellular encapsulation. Copyright (C) 1996 Elsevier Science Ltd.
\end{abstract}

Keywords-Antibacterial peptides; Insect immunity; Parasitoid wasps.

\section{Introduction}

The humoral immune molecules in insects include antibacterial and antifungal peptides, proteases, prophenoloxidase/pheno-

Address correspondence to $\mathrm{Dr}$ Bruno Lemaitre, UPR 9022, Institut de Biologie Moléculaire et Cellulaire, 15 rue René Descartes, 67084 Strasbourg Cedex, France. loxidase, and lectins (1-3). The celluiar immune reactions consist essentially of phagocytosis and encapsulation by circulating blood cells which are frequently mobilized to form melanotic capsules around foreign entities that are too large to be phagocytosed (4-6). The mechanism leading to the cellular encapsulation of parasites has been well documented at the morphological level (6) but is still poorly understood at the molecular level (7). The inducible antimicrobial peptides that appear in the hemolymph of insects in response to bacterial or fungal challenge are synthesized primarily by the fat body and hemocytes. In Drosophila, the genes or cDNAs encoding some of these inducible molecules have been cloned (i.e. cecropins $(8,9)$; diptericin $(10)$; defensin (11); drosocin (12); attacin (13); drosomycin (14)). The mechanism(s) controlling the expression of these antimicrobial peptides after immune challenge is an important questions in the field of insect immunology.

The objective of this investigation was to examine the extent to which mechanisms regulating the production of antibacterial peptides and cellular immune components in Drosophila melanogaster are co-regulated. To study the possible involvement of antibacterial genes in cellular encapsulation we monitored the 
inducible expression of two antibacterial peptides and one antifungal peptide in Drosophila melanogaster infected by the wasp parasitoids Leptopilina boulardi, Leptopilina heterotoma, or Trichopria sp., which oviposit a single egg into the body cavity of Drosophila larvae or pupae, respectively.

\section{Materials and Methods}

\section{Drosophila Stocks}

Oregon $\mathbf{R}$ flies were used as a standard wild-type strain. The transgenic strain, Dipt2.2-lacZ:1 is a ry ${ }^{506}$ C.S. line carrying a diptericin reporter gene on the $\mathrm{X}$ chromosome (10). The fusion gene containing $2.2 \mathrm{~kb}$ of diptericin upstream sequences fused to the bacterial lacZ coding region was inserted into the Carnegie 20 vector (15). The developmental and inducible expression of the Dipt2.2-lacZ transgene has been previously described (10). The inducible expression of the Dipt2.2-lacZ is superimposable to that of the resident diptericin gene at the end of the third larval stage.

All experiments were performed at $25^{\circ} \mathrm{C}$ unless otherwise stated.

\section{Origin of Wasp Strains and Infection Procedures}

The parasitic wasps $L$. boulardi, $L$. heterotoma, and Trichopria sp. used in this study were raised at $25^{\circ} \mathrm{C}$ on a wildtype strain of $D$. melanogaster (Oregon R). Leptopilina species lay their eggs inside Drosophila larvae. The egg hatches after approximately $48 \mathrm{~h}$ and the young wasp larva develops to the adult stage and emerges as an adult from the host puparium after 18-20 days. Trichopria lay eggs inside 2-day-old pupae and emerge as adults after 18 days.

Drosophila females were provided a 6-h oviposition period on standard medium. Second stage Drosophila larvae (approximately $50 \mathrm{~h}$ ) were exposed for $8 \mathrm{~h}$ to Leptopilina females that had not previously oviposited (4). To ensure adequate numbers of parasitized larvae and to minimize multiparasitism, five female parasitoids were used per 200-300 larvae. Under these conditions, $80 \%$ of the larvae were infected. From 48 to $72 \mathrm{~h}$ after parasitization, larvae were collected and divided into two batches. The first group was dissected to determine the percentage of parasitization, and the second group was used to evaluate gene expression. A similar procedure was followed when 2-4-day-old pupae were exposed to Trichopria.

\section{Injury Experiments}

Injury experiments were performed by pricking flies with a needle that had been previously dipped in a concentrated bacterial culture of Escherichia coli and Micrococcus luteus. The majority of bacterially challenged larvae died during the pupal stage.

\section{Quantitative Measurement of $\beta$-Galactosidase Activity}

The procedure described by Lemaitre and Coen (16) was applied to homogenates made from groups of five individuals of either third stage larvae or pupae. Results are given in nanomoles of product formed $/\left(\operatorname{min~} \mathrm{mg}^{-1}\right)$ protein.

\section{$\beta$-Galactosidase Localization}

The fat bodies were fixed for $5 \mathrm{~min}$ in PBS, pH 7.5, containing $1 \%$ glutaraldehyde and $1 \mathrm{mM} \mathrm{MgCl}_{2}$ (17). Subsequently, tissues were washed in PBS and immersed in $0.2 \%$ 5-bromo-4-chloro-3indolyl- $\beta$-D-galactopyranoside (X-gal), 


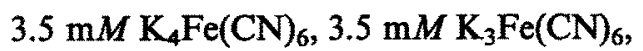
$1 \mathrm{~m} M \mathrm{MgCl}_{2}, 150 \mathrm{~m} M \mathrm{NaCl}, 10 \mathrm{~m} M$ $\mathrm{Na}_{2} \mathrm{HPO}_{4}, 10 \mathrm{mM} \mathrm{NaH} \mathrm{PO}_{4}$ and incubated for $4 \mathrm{~h}$ at $37^{\circ} \mathrm{C}$. The tissues were then mounted in glycerol.

\section{RNA Expression of Genes Encoding Antimicrobial Peptides}

Total RNA extraction and Northern blotting experiments were performed with tissues removed from control, bacteriachallenged, and parasitized Drosophila using methods described previously (18). The following probes were used to detect RNA expression: diptericin cDNA (19), drosomycin cDNA (14), rp49 cDNA, (a PCR fragment of approximately $400 \mathrm{bp}$ generated between two oligonucleotides designed after the rp49 coding sequence (20)) and a 21-mer oligonucleotide ( $5^{\prime}$ GATTCCCAGTCCCTGGATTGT-3') complementary to part of the coding sequence of cecropin A1 which is identical for cecropin A2 (8).

\section{Results}

Analysis of the Expression of a Diptericin-lacZ Reporter Gene in Transgenic Parasitized Drosophila

For our experiments we have used the transgenic Dipt2.2-lacZ:1 strain, which has stably integrated a lacZ reporter gene fused to the promoter $(2.2 \mathrm{~kb})$ of the gene encoding the antibacterial peptide diptericin. A previous study has shown that the induction of this reporter by immune challenge parallels that of the resident diptericin gene (10). In initial experiments the transgenic strain Dipt2.2lacZ:1 was found to be totally susceptible to the parasitoids, there being no evidence of a successful cellular encapsulation response against either species of Leptopilina or Trichopria (data not shown).
We have first investigated the expression of the Dipt-lacZ reporter gene in larvae infected by Leptopilina boulardi and Leptopilina heterotoma. For this, second stage Drosophila larvae carrying the transgene were parasitized and assayed for $\beta$-galactosidase activity $96 \mathrm{~h}$ post-infection (Table 1). In contrast to non-parasitized bacteria-challenged larvae, no expression of the Dipt2.2lacZ reporter gene was observed in infected larvae. Thus, neither $L$. boulardi nor $L$. heterotoma elicited a marked Dipt2.2lacZ: 1 expression.

To exclude the possibility that the absence of expression of the Dipt2.2lac $Z: 1$ transgene in wasp-infected second instar larvae reflected the low level of inducibility of the reporter gene at this stage of development (10), we have parasitized older, third stage larvae (approximately $96 \mathrm{~h}$ ) with $L$. boulardi. At 6-h post-infection, the fat body was removed from these hosts and stained for $\beta$-galactosidase activity (X-gal staining). Little or no $\beta$-galactosidase activity was observed in parasitized larvae or in nonparasitized control larvae. In contrast, a deep blue coloration was apparent in fat body cells of parasitized larvae that were

Table 1. Dipt-lacz2.2 Activity in Wasp-Infected Larvae

\begin{tabular}{lcc}
\hline Larvae & $\begin{array}{c}\text { Number of } \\
\text { measurements }\end{array}$ & $\begin{array}{c}\beta \text {-galactosidase } \\
\text { activity }\end{array}$ \\
\hline$(-)$ & 7 & $1.6 \pm 1.4$ \\
$(+)$ & 8 & $83.0 \pm 28.5$ \\
L.h. $(-)$ & 8 & $1.1 \pm 1.6$ \\
L.h. $(+)$ & 8 & $92.2 \pm 41.3$ \\
L.b. $(-)$ & 8 & $1.3 \pm 0.8$ \\
L.b. $(+)$ & 8 & $107.6 \pm 58.5$ \\
\hline
\end{tabular}

Results are the number of measurements, mean and the confidency interval $(p<5 \%)$. Activity was measured in a pool of five control or waspinfected wandering larvae that were either unchallenged $(-)$ or sacrified $3 \mathrm{~h}$ after bacterial challenge (+). The endogenous level of $\beta$-galactosidase was obtained by measuring the $\beta$-galactosidase activity in ry ${ }^{506}$ sibs of the Dipt2.2-lacZ:1 strains, devoid of Dipt2.2-lacZ:1 insertion. It was $0.9 \pm 0.2(n=8)$. L.h.: L. heterotoma; L.b.: L. boulardi. 
subsequently challenged with bacteria (data not shown). In separate experiments, quantitative measurements of $\beta$ galactosidase activity were made of homogenates of five third instar parasitized and bacteria-challenged larvae. These experiments showed levels of reporter gene expression that were similar to those obtained from non-parasitized, bacteria-challenged larvae (see Table 1). These observations indicate that parasitized larvae are not physiologically compromised and are fully capable of eliciting a wild-type induction of the diptericin reporter gene in response to bacterial challenge.

We next studied the expression of the diptericin reporter gene in Drosophila pupae parasitized by Trichopria. For these investigations, 2-day-old pupae from the Dipt2.2-lacZ:1 stock were used as hosts and exposed to parasitoids for $8 \mathrm{~h}$. A low but significant level of reporter gene expression was observed $24 \mathrm{~h}$ after Trichopria infection (Table 2). However, the level of Dipt2.2-lacZ:1 expression was significantly lower and induction kinetics slower than those observed in non-parasitized, bacteria-challenged pupae. In

\section{Table 2. Dipt2.2-lacZ:1 Activity In Trichopria- Infected Pupae}

\begin{tabular}{lcc}
\hline Pupae & $\begin{array}{c}\text { Number of } \\
\text { measurements }\end{array}$ & $\begin{array}{c}\beta \text {-galactosidase } \\
\text { activity }\end{array}$ \\
\hline$(-)$ & 13 & $2.6 \pm 2.6$ \\
$(+)$ & 8 & $51.5 \pm 18.9$ \\
6 h P.I. (-) & 8 & $15.5 \pm 6.4$ \\
14 h P.I. (-) & 8 & $11.5 \pm 5.5$ \\
14 h P.I.(+) & 8 & $57.8 \pm 20.0$ \\
24 h P.I.(-) & 8 & $19.7 \pm 6.7$ \\
48 h P.I.(-) & 8 & $13.6 \pm 6.8$ \\
\hline
\end{tabular}

Results are the number of measurements, mean and the confidency interval $(p<5 \%)$. Activity was measured in a pool of five control or wasp-infected pupae that were either unchallenged $(-)$ or sacrified $6 \mathrm{~h}$ after bacterial challenge $(+)$. The endogenous level of $\beta$ galactosidase was obtained by measuring the $\beta$-galactosidase activity in ry ${ }^{506}$ sib pupae of the Dipt2.2-lacZ:1 strains, devoid of Dipt/acZ insertion. It was $5.4 \pm 1.3 \quad(n=4)$. P.I.: postinfection. addition, following bacterial challenge, the Dipt2.2-lacZ:1 reporter gene was found to be fully inducible in pupae infected by Trichopria (Table 2).

\section{RNA Expression of Genes Encoding Antimicrobial Peptides in Parasitized Drosophila}

Northern blot analysis was used to monitor the expression of various genes encoding antimicrobial peptides after infection by wasp parasitoids. RNAs from control, parasitized, and both parasitized and bacteria-challenged larvae or pupae were extracted. The Northern blot filter was successively probed for the diptericin, cecropin $\mathrm{A}$ and drosomycin genes. A probe corresponding to the rp49 gene which encodes a ribosomal protein, was also used as a control for the amount of RNA.

The genes encoding the antibacterial peptides diptericin or cecropin A were not expressed in unchallenged larvae and pupae (Fig. 1), but a detectable level of expression was monitored for the antifungal peptide drosomycin, corroborating previous published data in the literature $(8,10,14)$. High levels of expression were observed for all three immune genes in bacteria-challenged insects (Fig. 1). In agreement with the results obtained above with the transgenic strain, there was little or no expression of these immune genes in wasp-infected individuals. In parasite-infected larvae and pupae, there was considerable variation in gene expression. Cecropin A activity was not induced by any parasitoid. The diptcricin gene was cxpressed in individuals parasitized by $L$. boulardi and by Trichopria. The antifungal gene encoding drosomycin was induced by $L$. heterotoma and Trichopria. However, radioactivity measurements indicated that the level of induction after wasp-infection was always significantly lower than after challenge by bacteria [(Fig. 1), data not shown]. Wild- 


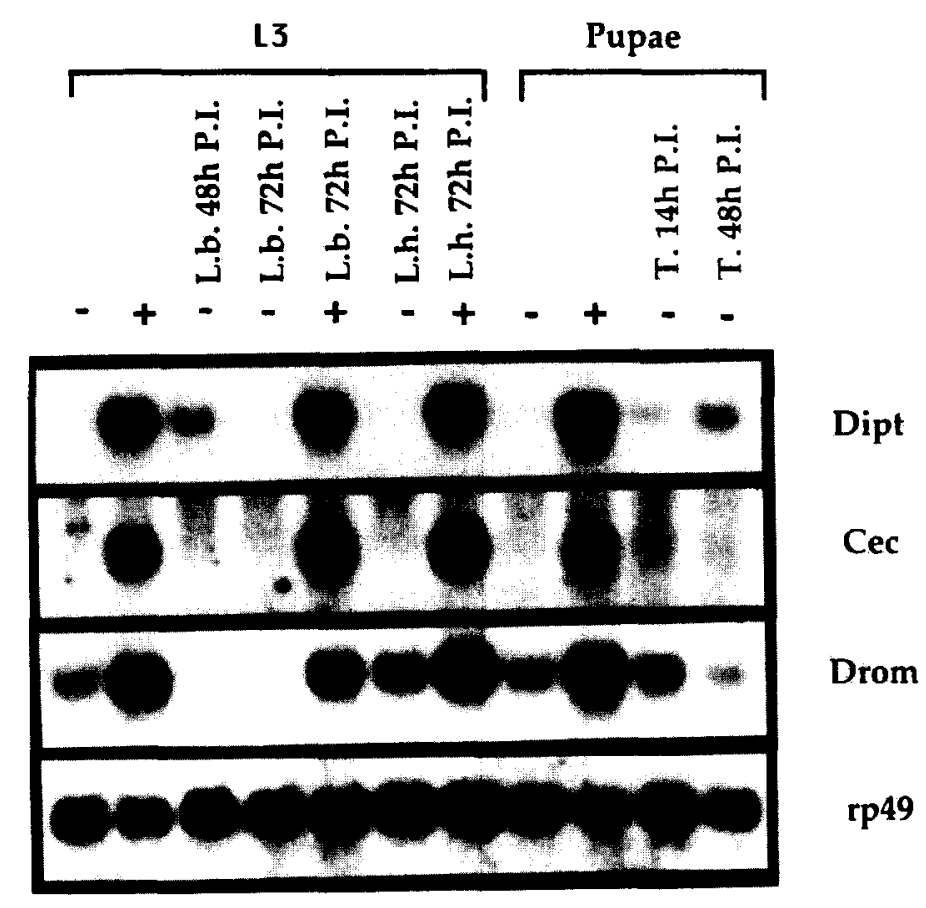

Figure 1. Transcriptional profiles of diptericin, cecropin and drosomycin in wild-type, parasitized and parasitized plus challenged larvae and pupae. Total RNA was extracted at different time intervals (as indicated) after parasitization from wild-type Oregon $^{R}$ larvae and pupae. Twenty microgram samples were fractionated by denaturing $1 \%$ agarose/formaldehyde gel electrophoresis, transferred onto a nylon membrane and successively hybridized with a nick-translated diptericin (Dipt) and drosomycin (Drom) cDNA probe; an end-labeled oligonucleotide probe complementary to cecropins A1 and A2 (CecA) transcripts and a nick-translated rp49 cDNA probe. -: unchallenged animals; +: $6 \mathrm{~h}$ challenged animals; L.b.: larvae parasitized by $L$. boulardi; L.h.: larvae parasitized by $L$. heterotoma; $T$ : pupae parasitized by Trichopria; L3, wandering third instar larva; P.I: post-infection.

type levels of induction of all three immune genes were exhibited in waspinfected larvae after bacterial challenge.

\section{Discussion}

Drosophila susceptible hosts were examined for evidence of parasitemediated induction of three genes encoding antimicrobial immune peptides (diptericin, cecropin $\mathrm{A}$, and drosomycin) following infection by either the larval $(L$. boulardi and L. heterotoma) or the pupal stage (Trichopria) parasitoids. We found no evidence for strongly induced antimicrobial immune responses in parasitized Drosophila using either specific $\beta$-galactosidase titration or Northern blotting techniques. These data indicate that eggs ovoposited in the larvae do not induce a full humoral antimicrobial response. Despite the low level of antimicrobial gene cxpression in wasp-infected Drosophila, the three antimicrobial genes remained fully inducible and were expressed upon subsequent bacterial challenge. These observations strongly suggest that the eggs ovoposited in the larvae are not recognized by the host's humoral mechanism. In contrast, it has been shown that in response to wasp infection, larvae elicit a cellular response (reviewed in Ref. 21). Rizki and Rizki showed that infection by Leptopilina wasps induces lamellocyte differentiation in larvae (22). Lamellocyte are large flattened hemocytes derived from plasmatocytes which are involved in the formation of the capsule (21). This last observation indicates that 
the presence of the parasitoid is recognized by the immune system of the host. This cellular response is, however, later selectively incapacited in susceptible larvae (23). Indeed as shown for $L$. heterotoma, the number of lamellocytes from infected larvae decreased, probably due to a destructive factor derived from the accessory gland of the female wasp's reproductive system (24). Taken together, the results of this study indicate that, while altering the cellular response, infection of Drosophila by wasp parasitoids does not affect the host's humoral immune mechanism. This last result suggests that the humoral and cellular immune mechanisms may be activated by different mechanisms.

The observations made in this study using two species of Leptopilina support in part the recent investigations of Coustau et al. (25) who likewise were unable to detect the induction of antibacterial peptides in immune-reactive (resistant) Drosophila infected with $L$. boulardi. This result demonstrates that the presence of antimicrobial peptides is not required for successful encapsulation of the parasitoid (25). However, these authors detected an elevated antibacterial activity in susceptible hosts infected by $L$. boulardi. (25). In contrast, with the three different parasitoid species tested here, only a low level of antimicrobial expression in both parasitized Drosophila larvae and pupae was observed. This was attributed to bacterial contamination which occurred during parasitoid oviposition. The combined data suggest that infection of Drosophila by wasp parasitoids does not diminish the host's antimicrobial immune response, and that this humoral mechanism is regulated independently of the host's cellular immune system. The existence of different recognition mechanisms does not preclude that the cellular and humoral responses share common regulatory elements. This was recently suggested by two reports indicating that rel proteins and the Toll receptor may be involved in both the control of antimicrobial genes and lamellocyte differentiation $(18,26)$.

As with other organisms, insects have evolved with different adaptative responses to various types of aggression (i.e. infection by metazoan parasitoids, bacteria, or fungi). The results of this investigation suggest that, in Drosophila, the humoral response elicited by microbial agents is regulated by mechanisms that differ from those which control cellular encapsulation. Additional evidence to support this proposal comes from recent studies showing the differential induction of antibacterial and antifungal genes (27). An important goal of current research in the field of insect immunity is to decipher the specific recognition mechanism(s) for pathogens that trigger the immune defense.

Acknowledgements-The authors are indebted to $\operatorname{Pr}$ Michel Boulétreaux for sending parasitoid wasps and to Dr Marie Meister. The technical assistance of Reine Klock and Raymonde Syllas is gratefully acknowledged. Anthony Nappi is grateful for the assistance provided by the National Science Foundation (IBN 950 4796), the Research Services of Loyola University Chicago, and to Emily Vass of the Department of Biology at Loyola University Chicago. Emmanuelle Nicolas and Bruno Lemaitre acknowledge support from the Centre National de la Recherche Scientifique, the University Louis Pasteur. They thank Pr Jules Hoffmann for his interest in this study.

\section{References}

1. Ashida, M.; Yamazaki, H. I. Biochemistry of the phenoloxidase system in insects: with special reference to its activation. In Ohnishi, E;
Ishizaki, H., Eds Molting and metamorphosis. Tokyo/Berlin: Japan Sci. Soc. Press/Springer; 1990: 239-265. 
2. Huitmark, D. Immune reactions in Drosophila and other insects: a model for innate immunity. Trends Genet. 9:178-183; 1993.

3. Hoffmann, J. A. Innate immunity of insects. Curr. Opin. Immunol. 7:4-10; 1995.

4. Carton, $Y_{;}$Frey, F.; Nappi, A. J. Inheritance of cellular immune resistance in Drosophila melanogaster. Heredity 69:393-399; 1992.

5. Nappi, A. J.; Vass, E. Melanogenesis and the generation of cytotoxic molecules during insect cellular immune reactions. Pigment Cell Res. 6:117-126; 1993.

6. Vass, E.; Nappi, A. J.; Carton, Y. Comparative study of immune competence and host susceptibility in Drosophila melanogaster parasitized by Leptopilina boulardi and Asobara tabida. J. Parasitol. 79:106-112; 1993.

7. Kobayashi, M.; Johansson, M. W.; Söderhäll, $\mathrm{K}$. The $76 \mathrm{kD}$ cell-adhesion factor from crayfish haemocytes promotes encapsulation in vitro. Cell. Tissue Res. 260:13-18; 1990.

8. Kylsten, P.; Samakovlis, C.; Hultmark, D. The cecropin locus in Drosophila; a compact gene cluster involved in the response to infection. EMBO J. 9:217-224; 1990.

9. Tryselius, Y.; Samakovlis, C.; Kimbrell, D. A.; Hultmark, D. CecC, a cecropin gene expressed during metamorphosis in Drosophila pupae. Eur. J. Biochem. 204:395-399; 1992.

10. Reichhart, J. M.; Meister, M.; Dimarcq, J. L.; Zachary, D.; Hoffmann, D.; Ruiz, C.; Richards, G.; Hoffmann, J. A. Insect immunity: developmental and inducible activity of the Drosophila diptericin promoter. EMBO J. 11:1469-1477; 1992.

11. Dimarcq, J. L.; Hoffmann, D.; Meister, M.; Bulet, P.; Lanot, R.; Reichhart, J. M.; Hoffmann, J. A. Characterization and transcriptional profiles of a Drosophila gene encoding an insect defensin. A study in insect immunity. Eur. J. Biochem. 221:201-209; 1994.

12. Bulet, P.; Dimarcq, J. L; Hetru, C.; Lagueux, M.; Charlet, M.; Hegy, G.; Van Dorsselaer, A.; Hoffmann, J. A. A novel inducible antibacterial peptide of Drosophila carries an O-glycosylated substitution. J. Biol. Chem. 268:14893-14897; 1993.

13. Asling, B.; Dushay, M. S.; Hultmark, D. Identification of early genes in the Drosophila immune response by PCR-based differential display: the Attacin A gene and the evolution of attacin-like proteins. Insect Biochem. Mol. Biol. 25:511-518; 1995.

14. Fehlbaum, P.; Bulet, P.; Michaut, L.; Lagueux, M.; Broekaert, W. F.; Hetru, C.; Hoffmann, J. A. Insect immunity: septic injury of Drosophila induces the synthesis of a potent antifungal peptide with sequence homology to plant antifungal peptides. J. Biol. Chem. 269:31159. $31163 ; 1994$.
15. Rubin, G. M.; Sprading, A. C. Genetic transformation of Drosophila with transposable element vectors. Science 218:348-353; 1982.

16. Lemaitre, B.; Coen, D. P. Regulatory products repress in vivo the $P$ promoter activity in $P$ LacZ fusion genes. Proc. Natl. Acad. Sci. U.S.A. 88:4419-4423; 1991 .

17. Hiromi, Y; Kuroiwa, A; Gehring, W. J. Control of the Drosophila segmentation gene fushi tarazu. Cell 43:603-614; 1985.

18. Lemaitre, B.; Meister, M.; Govind, S.; Georgel, P.; Steward, R.; Reichhart, J. M.; Hoffmann, J. A. Functional analysis and regulation of nuclear import of dorsal during the immune response in Drosophila. EMBO J. 14:536-545; 1995.

19. Wicker, C; Reichhart, J. M.; Hoffmann, D; Hultmark, D.; Samakovlis, C.; Hoffmann, J. A. Insect immunity. Characterization of a Drosophila cDNA encoding a novel member of the diptericin family of immune peptides. J. Biol. Chem. 265:22493-22498; 1990.

20. O'Connell, P.; Rosbash, M. Sequence, structure and codon preference of the Drosophila ribosomal protein 49 gene. Nucleic Acid Res. 12:5495-5513; 1984.

21. Rizki, T.M.; Rizki, R.M. Parasitoid-induced cellular immune deficiency in Drosophila. In: Beck, G. Ed. Primordial immunity. Foundations for the vertebrate immune system. Conference, Woods Hole, Massachusetts, U.S.A. May 2-5 1993.

22. Rizki, T. M.; Rizki, R. M. Lamellocyte differentiation in Drosophila parasitized by Leptopilina. Dev. Comp. Immunol. 16:103; 1993-110.

23. Rizki, T. M; Rizki, R. M.; Carton, Y. Leptopilina heterotoma and $L$. boulardi: strategies to avoid cellular defense responses of Drosophila melanogaster. Exp. Parasitol. 70:466-475; 1990.

24. Rizki, T. M.; Rizki, R. M. Selective destruction of a host blood cell type by a parasitoid wasp. Proc. Natl. Acad. Sci. U.S.A. 81:6154-6158; 1984.

25. Coustau, C.; Carton, Y.; Nappi, A. J.; Shotkoski, F; French-Constant, R. Differential induction of antibacterial transcripts in Drosophila susceptible and resistant to parasitism by Leptopilina boulardi. Insect Biochem. Mol. Biol. (in press).

26. Goving, S. Rel signalling pathway and the melanotic tumor phenotype of Drosophila. Biochemical Society Transactions 24:39-44; 1996.

27. Lemaitre, B.; Kromermetzger, E.; Michaut, L; Nicolas, E.; Meister, M.; Georgel, P.; Reichart, J. M.; Hoffmann, J. A. A recessive mutation, immune deficiency ( $i m d)$, defines two distinct control pathways in Drosophila host defense. Proc. Natl. Acad. Sci. U.S.A. 92:9465-9469; 1995. 